\title{
Distant Ureteral Metastasis from Colon Adenocarcinoma: Report of a Case and Review of the Literature
}

\author{
Ferakis Nikolaos, ${ }^{1}$ Anastasopoulos Panagiotis, ${ }^{1}$ Bouropoulos Konstantinos, ${ }^{1}$ \\ Samaras Vassilios, ${ }^{2}$ and Poulias Iraklis ${ }^{1}$ \\ ${ }^{1}$ Department of Urology, Korgialenio-Benakio Hellenic Red Cross Hospital, Athanasaki 1, 11526 Athens, Greece \\ ${ }^{2}$ Department of Pathology, Korgialenio-Benakio Hellenic Red Cross Hospital, Athanasaki 1, 11526 Athens, Greece \\ Correspondence should be addressed to Ferakis Nikolaos; ferakis@otenet.gr
}

Received 22 November 2013; Accepted 21 January 2014; Published 4 March 2014

Academic Editors: B. Detti, J. Park, and C. Pavlovich

Copyright (c) 2014 Ferakis Nikolaos et al. This is an open access article distributed under the Creative Commons Attribution License, which permits unrestricted use, distribution, and reproduction in any medium, provided the original work is properly cited.

Carcinomas arising from organs neighbouring the ureter can directly infiltrate the ureter. Distant ureteral metastasis from colon adenocarcinoma is extremely rare and usually an incidental finding in performed autopsies. We report a case of a right ureteral metastasis in a 65 -year-old Caucasian male with a history of rectal cancer for which he had been treated 4 years before. He presented with asymptomatic moderate right hydronephrosis. The patient underwent a right nephroureterectomy. Histology of the ureter revealed transmural adenocarcinoma with infiltration of the mucosa. Infiltration of the muscular coat of the bladder was found 2 years later. Thus, cystectomy and left ureterocutaneostomy were performed. The patient died 6 months later due to toxic megacolon during chemotherapy. The differential diagnosis of ureteral adenocarcinoma, especially in patients with previous history of colon adenocarcinoma, should include the possibility of distant metastasis from the primary colonic tumor.

\section{Introduction}

Distant metastases to the ureter may occur through lymphatic and/or blood vessels excluding ureteral involvement by direct extension [1-3]. These metastases have been reported more commonly arising from stomach, breast, lung, cervix, prostate, pancreas, and lymphoma [2-6]. Distant ureteral metastases from colon adenocarcinoma are considered to be very rare [2-10]. We present a case of this condition and review the English language literature.

\section{Case Report}

In November 2005 a 61-year-old male patient underwent rectosigmoidectomy for nonmetastatic rectal cancer (grade II, stage T3N1MO), which was followed by chemotherapy (oxaliplatin and capecitabine) and radiotherapy. He had no signs of relapse during the follow-up. In May 2009 an asymptomatic moderate right hydronephrosis in followup computed tomography (CT) scan was revealed. The ureteropyelogram (Figure 1) showed a $1.5 \mathrm{~cm}$ filling defect in the lower right ureter. Ureteroscopy revealed a papillary lesion. Urine cytology showed malignant cells strongly suspicious for adenocarcinoma which correlated with the biopsy findings where immunohistochemistry demonstrated carcinoma with glandular differentiation and positivity for cytokeratin (CK) 20, fully compatible with a metastatic colonic type adenocarcinoma. The patient was fully evaluated with colonoscopy and CT (Figure 2) with no signs of local or distant relapse.

The patient underwent right nephroureterectomy. Histology revealed intraluminal adenocarcinoma (grade II) with infiltration of the mucosa, muscular layer, and adventitia (Figure 3). Periureteral tissue was free of tumor. This was followed by 6 cycles of chemotherapy. The patient was closely followed up afterwards until February 2012, where infiltration of the muscular coat of the bladder by adenocarcinoma (CK7-, CK20+), without other metastases, was found.

In March 2012 radical cystectomy and extended pelvic lymphadenectomy with left ureterocutaneostomy were performed. Foci of a moderately differentiated adenocarcinoma were identified in the bladder wall, localized both in the 


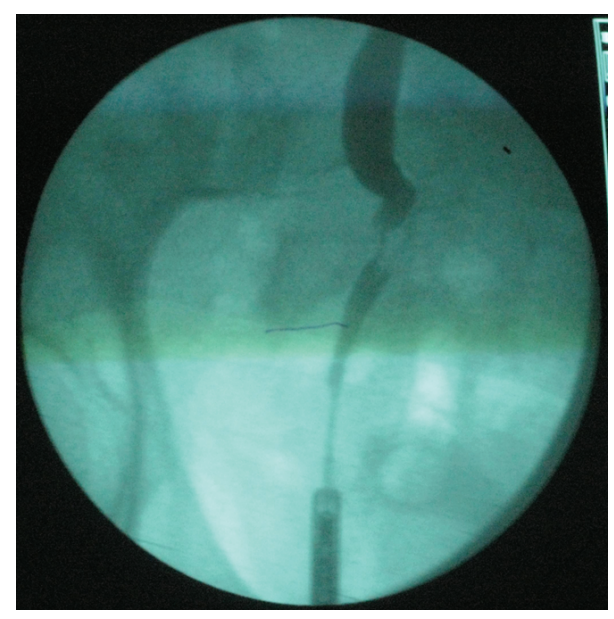

FIGURE 1: Ureteropyelogram revealing an obstruction of the lower right ureter, local ureteral dilatation, and intraluminal filling defect.

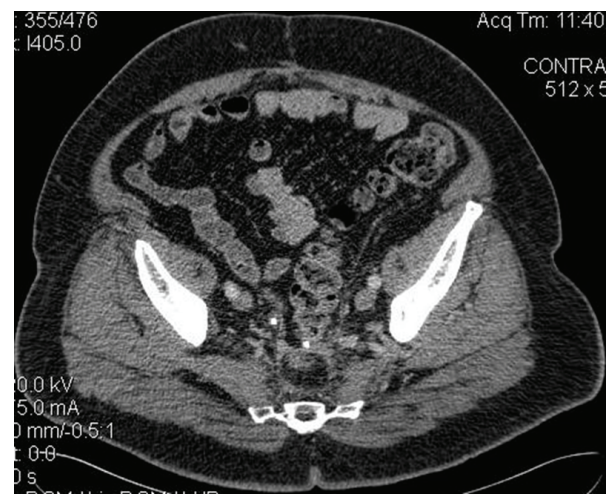

FIGURE 2: Computed tomography of the pelvis showing no evidence of extraureteral relapse.

lamina propria as well as in the perivesical adipose tissue. Neoplastic vascular plugs and carcinomatous perineural infiltration were also found. The neoplastic population was immunohistochemically positive for CK7, CK20, carcinoembryonic antigen (CEA), $\beta$-catenin, and $\mathrm{p} 53$. The surgical margins were free of neoplastic disease. Severe lymphatic inflammatory reaction without evidence of lymph node metastasis was found (pT3apNO). The patient died 6 months later due to toxic megacolon during chemotherapy.

\section{Discussion}

Distant ureteral metastasis from primary colon adenocarcinoma was first reported in 1936 [2]. The most common sites of primary lesion are the breast, stomach, lung, cervix, prostate, pancreas, and lymphoma [2-6]. Reviewing the English language literature, reports describing true ureteral metastases from colon cancer are scarce [2-10]. Twenty-three cases have been published worldwide so far (Table 1).

In the first half of the last century, these ureteral metastases have been only described as incidental findings during autopsy due to the fact that the majority of patients

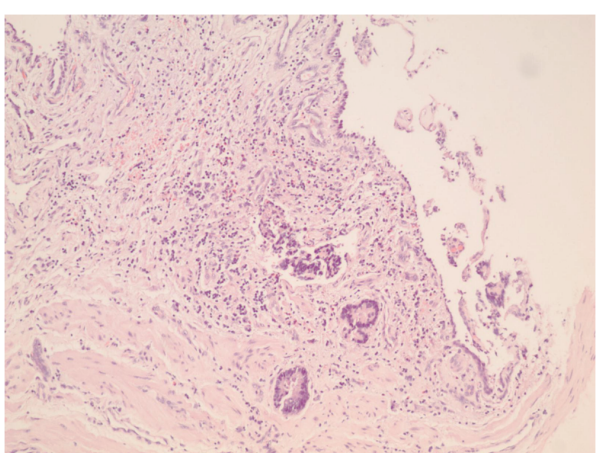

FIGURE 3: Neoplastic cellular population with an adenocarcinomatous pattern infiltrates the lamina propria of the ureter (hematoxylin/eosin $\times 10$ ). Note the overlying atrophic or inflamed urothelium.

were asymptomatic [2-6]. MacLean and Fowler reported an incidence of 2 cases in 10.223 performed autopsies [4]. Symptomatic patients most frequently described low back pain, renal colic, and/or anuria in cases of bilateral ureteral obstruction $[5,7,8]$. The fact that hematuria is a rather uncommon finding can be explained considering that ulceration of the ureteral mucosa is not likely to occur $[2,3,6]$.

There are two interesting parameters that must be emphasized. First, in the majority of cases, there were synchronous metastatic lesions in other organs or lymph nodes (Table 1). In our case, a single ureteral metastasis with no evidence of other metastatic lesions was found 4 years after primary rectal adenocarcinoma and metachronous bladder adenocarcinoma developed 2 years later. Second, in our patient the ureteral lesion occupied not only the muscularis and the adventitia but the mucosa as well. According to the literature, mucosal involvement is extremely rare, with only 3 definite cases published so far $[7,9,10]$.

Metastatic tumour cell deposition in the ureter has been described as infiltration of the periureteral soft tissue with compression to the ureteral wall, transmural infiltration, or, uncommonly, infiltration of the local mucosa $[2,3]$. The precise mechanism responsible for distant ureteral metastasis and the factors promoting this process are still quite unknown. MacKenzie and Ratner were the first to propose as rigid criteria for the diagnosis of distant ureteral metastasis the finding of malignant cells in the perivascular lymphatic spaces or in the blood vessels around the ureter [1]. Later, Presman and Ehrlich modified the criterion as the demonstration of malignant cells in a portion of the ureteral wall together with the absence of any neoplasm in adjacent tissues [2]. Our patient showed no tumoral lesions in the periureteral and retroperitoneal space during surgery indicating that the disease was true distant ureteral metastasis from the colon according to the criterion of Presman and Ehrlich that has been widely accepted and used to date.

Metastatic ureteral adenocarcinoma after primary colon adenocarcinoma is aggressive and has a poor prognosis without evidence of long-term survival in the reported cases [210]. Due to the rarity of the disease there are no standardized 


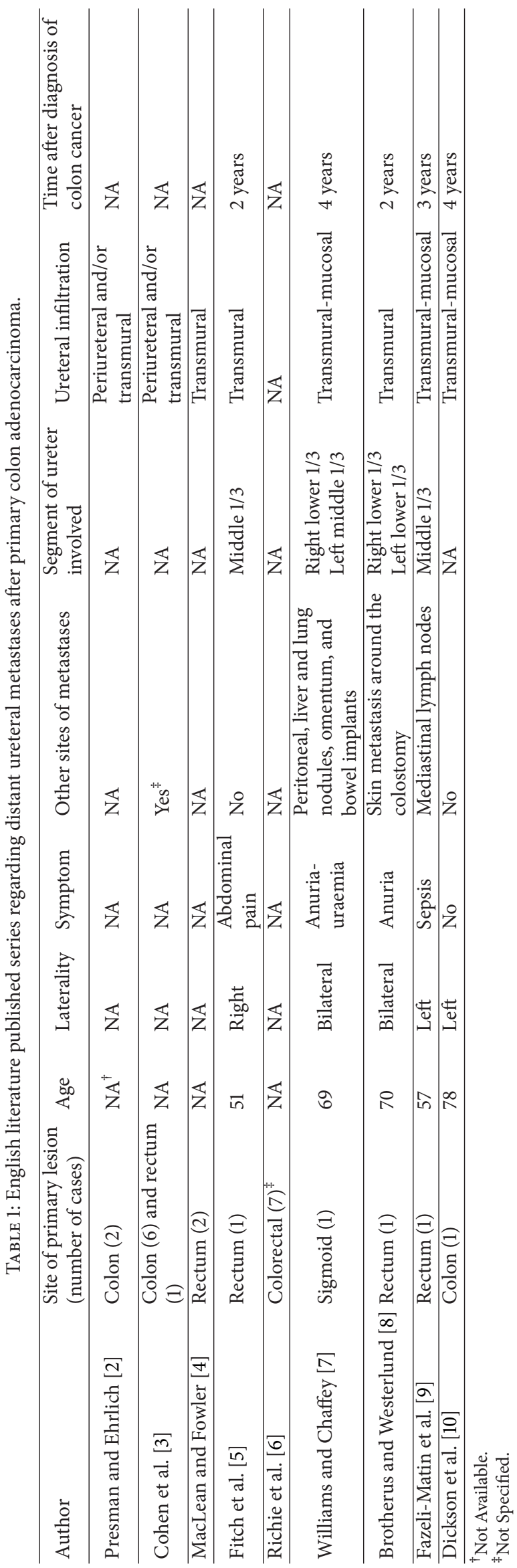


treatment protocols but chemotherapy remains mainstay in combination with the surgical treatment.

In conclusion, the colon is a very rare site of origin for distant ureteral metastasis. Although this condition is infrequently encountered in clinical practice, the possibility of metastatic ureteral carcinoma must be considered in the differential diagnosis, once there is a clinical or radiographic evidence of ureteral obstruction in a patient, who has a history of colon cancer regardless the stage and previous therapy.

\section{Conflict of Interests}

The authors declare that there is no conflict of interests regarding the publication of this paper.

\section{References}

[1] D. W. MacKenzie and M. Ratner, "Metastatic growths in the ureter. A report of three cases and a brief review of the literature," Canadian Medical Association Journal, vol. 25, no. 3, pp. 265-270, 1931.

[2] D. Presman and L. Ehrlich, "Metastatic tumors of the ureter," Journal of Urology, vol. 59, pp. 312-325, 1948.

[3] W. M. Cohen, S. Z. Freed, and J. Hasson, "Metastatic cancer to the ureter: a review of the literature and case presentations," Journal of Urology, vol. 112, no. 2, pp. 188-189, 1974.

[4] J. T. MacLean and V. B. Fowler, "Pathology of tumors of the renal pelvis and ureter," Journal of Urology, vol. 75, pp. 384-415, 1956.

[5] W. P. Fitch, J. R. Robinson, and H. M. Radwin, "Metastatic carcinoma of the ureter," Archives of Surgery, vol. 111, no. 8, pp. 874-876, 1976.

[6] J. P. Richie, G. Withers, and R. M. Ehrlich, "Ureteral obstruction secondary to metastatic tumors," Surgery Gynecology and Obstetrics, vol. 148, no. 3, pp. 355-357, 1979.

[7] D. F. Williams and B. T. Chaffey, "Metastatic adenocarcinoma of the sigmoid colon masquerading as bilateral intraluminal ureteral papillomas," British Journal of Urology, vol. 38, no. 5, pp. 563-566, 1966.

[8] J. V. Brotherus and R. M. Westerlund, "Metastatic carcinoma of the ureter. A report of three cases," Scandinavian Journal of Urology and Nephrology, vol. 5, no. 1, pp. 86-90, 1971.

[9] S. Fazeli-Matin, H. S. Levin, and S. B. Streem, "Ureteroscopic diagnosis of intraluminal metastatic rectal carcinoma," Urology, vol. 49, no. 6, pp. 955-956, 1997.

[10] B. C. Dickson, V. L. Fornasier, C. J. Streutker, and R. J. Stewart, "Ureteric obstruction: an unusual presentation of metastatic colon carcinoma," The Canadian Journal of Urology, vol. 14, no. 2, pp. 3526-3528, 2007. 


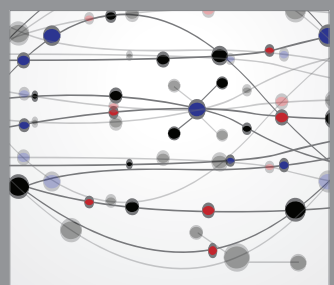

The Scientific World Journal
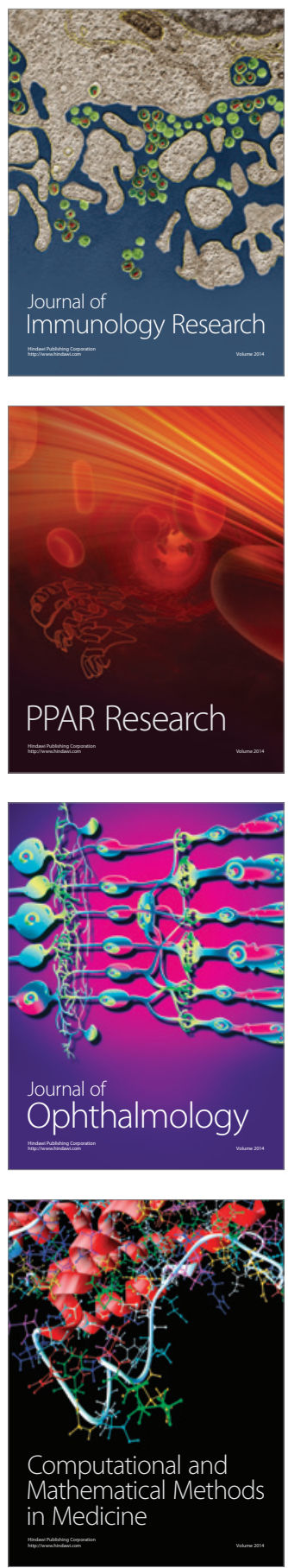

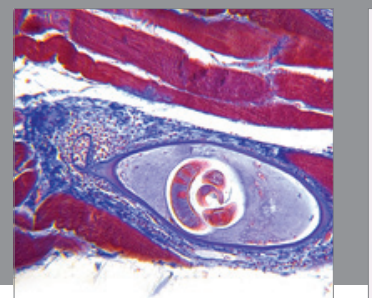

Gastroenterology

Research and Practice
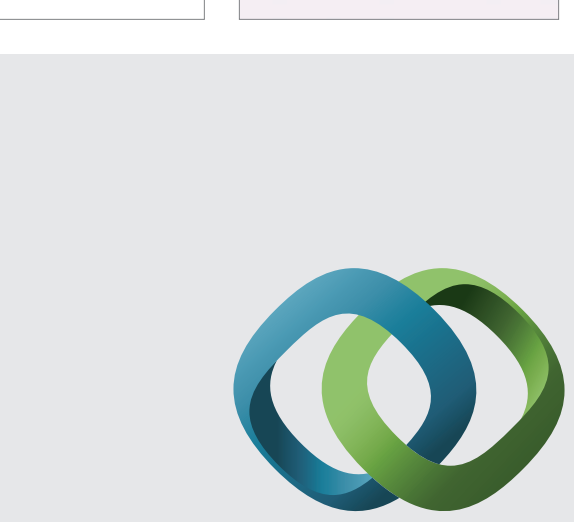

\section{Hindawi}

Submit your manuscripts at

http://www.hindawi.com
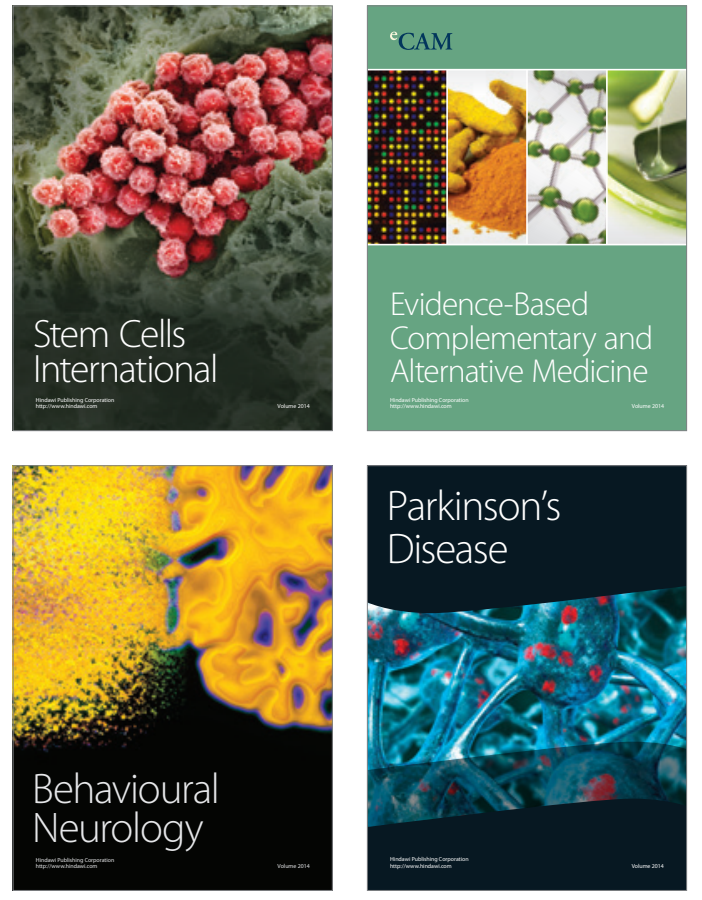
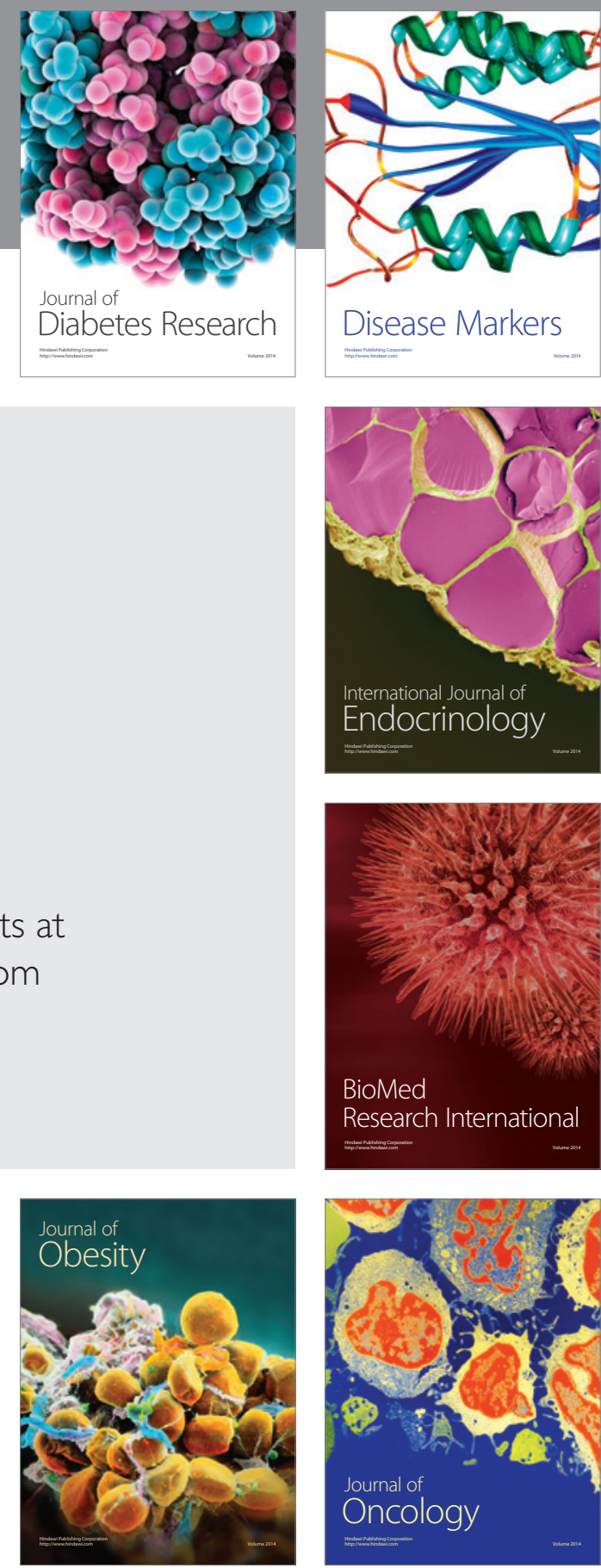

Disease Markers
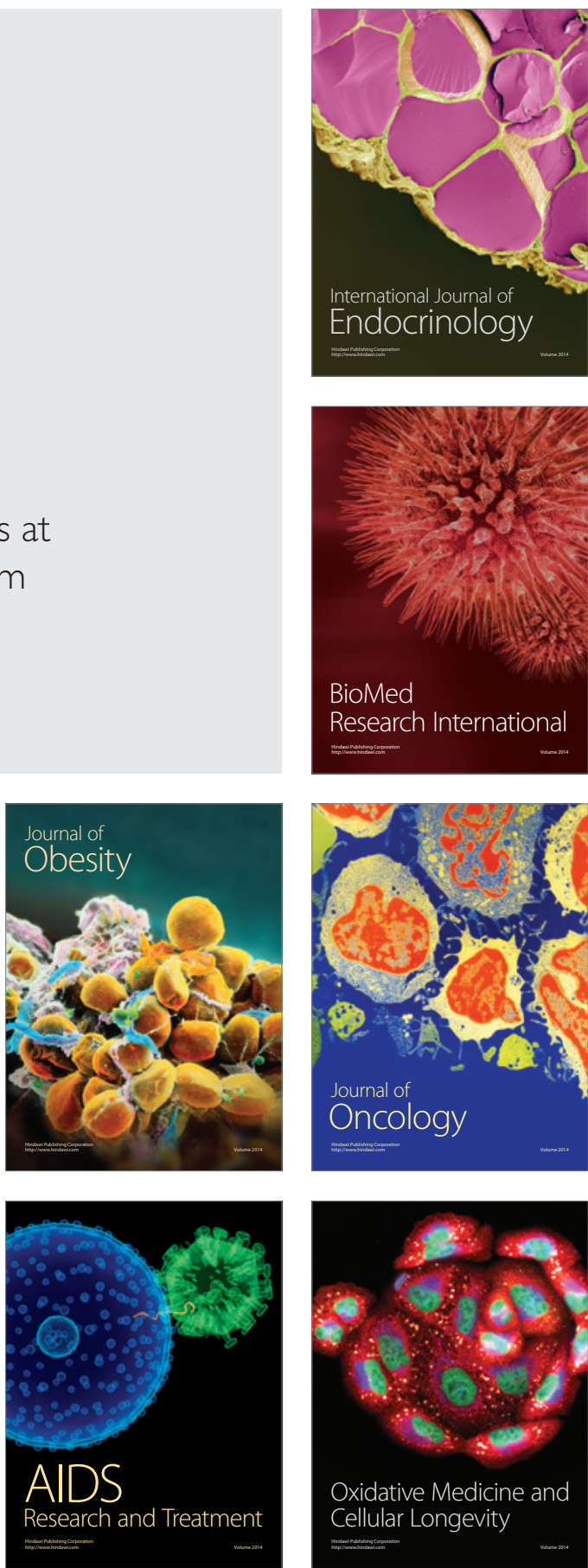